This document is confidential and is proprietary to the American Chemical Society and its authors. Do not copy or disclose without written permission. If you have received this item in error, notify the sender and delete all copies.

\title{
Rapid Fabrication of Metal-Organic Framework Films from Metal Substrates Using Intense Pulsed Light
}

\begin{tabular}{|r|l|}
\hline Journal: & Crystal Growth \& Design \\
\hline Manuscript ID & cg-2018-01145y.R1 \\
\hline Manuscript Type: & Article \\
\hline Date Submitted by the Author: & $\mathrm{n} / \mathrm{a}$ \\
\hline Complete List of Authors: & $\begin{array}{l}\text { Yim, Changyong; University of Calgary, Mechanical and Manufacturing } \\
\text { Engineering } \\
\text { Abuzalat, Osama; University of Calgary, Mechanical and Manufacturing } \\
\text { Engineering } \\
\text { Elsayed, Mohamed; University of Calgary, Mechanical and Manufacturing } \\
\text { Engineering; Military Technical College, Chemical Engineering } \\
\text { Park, Simon; Simon Park, Mechanical and Manufacturing Engineering } \\
\text { Kim, Seonghwan; University of Calgary, Mechanical and Manufacturing } \\
\text { Engineering }\end{array}$ \\
\hline
\end{tabular}

SCHOLARONE

Manuscripts 


\title{
Rapid Fabrication of Metal-Organic Framework Films from Metal Substrates Using Intense Pulsed Light
}

\author{
Changyong Yim $^{1 \uparrow *}$, Osama Abuzalat ${ }^{1 \dagger}$, Mohamed Elsayed ${ }^{1,2}, \operatorname{Simon}$ Park $^{1}$, Seonghwan Kim ${ }^{1, *}$ \\ ${ }^{1}$ Department of Mechanical and Manufacturing Engineering, University of Calgary, Calgary, Alberta T2N \\ 1N4, Canada \\ ${ }^{2}$ Department of Chemical Engineering, Military Technical College, Cairo, Egypt \\ ${ }^{\dagger}$ These authors equally contributed to this work. \\ *Corresponding author e-mail: Changyong Yim cy.yim1@gmail.com \& Seonghwan Kim \\ sskim@ucalgary.ca
}

\begin{abstract}
In this article, we demonstrate an innovative approach for the fabrication of uniform metal-organic framework (MOF) films on $\mathrm{Cu}$ or $\mathrm{Zn}$ metal substrates by using intense pulsed light (IPL). The metal substrates are first treated with a strong oxidizing agent to convert the metal to the corresponding metal hydroxide then MOF films are prepared by in-situ growth over the metal hydroxide surface with organic ligand by multiple IPL irradiations at room temperature and ambient condition. The metal hydroxide absorbs the light from IPL which discharges an excessive energy with relatively short pulse duration (milliseconds), then converts the light to thermal energy. Four well-known MOF compounds; $\mathrm{Cu}-\mathrm{BTC}, \mathrm{Cu}-$ BDC, ZIF-8, and MOF-5 film are successfully synthesized and characterized by scanning electron microscopy and x-ray diffraction analysis. The effects of organic ligand concentration and IPL exposure time on MOF film synthesis are systematically investigated. The innovative fabrication method presented
\end{abstract}


in this study offers many advantages such as short processing time, low cost process under ambient conditions, less use of consumable chemicals, and applicability to other MOF films fabrication. 


\section{Introduction}

Metal-organic frameworks (MOFs) and other so-called crystalline porous solids are of great scientific interest because of their valuable applications in gas sensing, storage, and separation [1-4]. The highly-ordered nature, tunable pore volume, ultrahigh porosity, and the ability to tailor the frameworks' chemical functionality by modifying the organic ligands provide great potentials [5-8]. Moreover, the creation of desired structural, chemical and physical properties in demand is possible depending on the chemical structures and properties of the ligands and attaching metals [9, 10]. Generally, MOFs are synthesized by the congregation of metal ions, small metal-containing clusters which act as junctions and organic ligands as linkers [11-13]. Most reported MOFs compounds have been synthesized by hydrothermal or solvothermal methods accompanied by comparatively high temperature $\left(100{ }^{\circ} \mathrm{C}-300{ }^{\circ} \mathrm{C}\right)$ and sometimes at increased pressure (above 1 bar) $[14,15]$. The formation reactions are sometimes carried out in a polytetrafluoroethylene (Teflon) reactor sealed by a stainless-steel autoclave [16-19]. However, these methods typically have the obvious disadvantage of requiring long reaction time (hours to weeks), depending upon the reaction temperature, reagent concentrations, the reaction solvent, and other factors $[20,21]$. While there are a number of articles reporting designing new types of MOFs [22-24], there are few reports on their emerging synthesis techniques. Recently, alternative synthesis methods, such as sonochemical [4], microwave heating [25, 26], electrochemical [27], and solvent-free synthesis [28, 29], have been reported. These alternative synthesis methods allowed the large-scale synthesis of MOFs with substantial reduction in crystallization time and also sometimes have great control on crystal size compared with conventional oven heating methods $[4,30]$. Although all the previous processes are straightforward methods to produce crystalline MOFs particles, it is not easy to prepare a thin film of MOFs on a solid substrate $[1,31]$. Generally, MOFs films have been prepared by several techniques including solvothermal synthesis procedures with immersion of the selected substrate into the precursor solutions of the particular 
MOFs material [32-35], layer by layer deposition (LBL) [36, 37], liquid phase epitaxy (LPE) [38], Langmuir Blodgett (LB) method [39] and electrochemical deposition [40]. However, the fabrication of MOFs films is challenging since the organic linkers commonly do not provide convertible linkage groups that can form bonds with functional groups on the surface of the supports [31]. There are several strategies to address this issue including; surface treatment and modification with organic functional groups and the use of electro-statically consistent substrates (e.g., $\mathrm{Al}_{2} \mathrm{O}_{3}$ ) [41, 42]. Especially, an exceptionally favorable case is achieved when the substrate is made of the same metal as the MOFs to be fabricated. When the substrate is a metal such as $\mathrm{Cu}$ or $\mathrm{Zn}$, in situ growth of MOFs film can be more effective because the metal substrate may act as a source of metal ions for the synthesis of the MOFs film.

In this paper, we demonstrate a novel method for synthesizing MOFs films, in a rapid and facile manner, on one side of metal substrate by the use of Intense Pulsed Light (IPL) (also called photonic light or flashlight) heating technique. This process can be performed at room temperature under ambient conditions. High-output flash-lamp is a high energy, non-laser light source that emits a broad wavelength output (from $300 \mathrm{~nm}$ to $1000 \mathrm{~nm}$ range) of noncoherent light. Light pulses are created by a blast of electric current through a xenon gas-filled cell [43]. The flash lamp pulses have a specific intensity and duration, allowing for a limited and controlled energy to be delivered into the synthesis process [43-46]. Flash lamp discharges high energy in milliseconds, which enables the chemical reaction between metal hydroxide on metal substrates and organic ligands. To prove the versatility of our method, four well-known MOFs compounds; $\mathrm{Cu}-\mathrm{BTC}, \mathrm{Cu}-\mathrm{BDC}, \mathrm{ZIF}-8$, and MOF-5 films are synthesized on either $\mathrm{Cu}$ or $\mathrm{Zn}$ substrate and characterized by scanning electron microscopy (SEM) and X-ray diffraction (XRD) analysis. These MOFs films are chosen because they have high porosity and surface area that have high potential for gas sensing applications. 


\section{Experimental Details}

\subsection{Materials}

Trimesic acid (1,3,5-benzene tricarboxylic acid $\left(\mathrm{H}_{3} \mathrm{BTC}\right), 98 \%$, Alfa Aesar), terephthalic acid (benzene1,4-dicarboxylic acid (BDC), 98+\%, VWR), 2-methylimidazole (2-MIM, 99\%, Sigma-Aldrich), N,Ndimethylformamide (DMF, 99.8\%, VWR), triethylamine (TEA, 99\%, Alfa Aesar), ammonium persulfate $\left(\left(\mathrm{NH}_{4}\right)_{2} \mathrm{~S}_{2} \mathrm{O}_{8}, 98 \%\right.$, Aldrich), sodium hydroxide ( $\mathrm{NaOH}, 97 \%$, VWR), acetone $(99 \%$, VWR), and distilled deionized water (DDW) are used without further purification in the synthesis of MOFs films. Copper-clad substrate (RS component code: 159-5773) and zinc strip (0.25 mm thick, 99.98\%, Alfa Aesar) are used as substrates in the MOFs film syntheses.

\subsection{Substrate preparation}

$\mathrm{Cu}-\mathrm{BTC}$ and $\mathrm{Cu}-\mathrm{BDC}$ are synthesized on copper-clad substrates, and ZIF-8 and MOF-5 films are synthesized on zinc strips. Prior to synthesis of MOFs films on the metal substrate, the substrates are sonicated for $20 \mathrm{~min}$ in a 1:1 mixture of ethanol and DDW to remove any contaminants. After being washed with copious amounts of DDW and acetone, the substrates are dried by blowing nitrogen. After cleaning the metal substrates, the metallic copper and zinc substrates are oxidized by immersing into an aqueous solution of $4 \mathrm{ml}$ sodium hydroxide $(\mathrm{NaOH}, 10 \mathrm{M}), 2 \mathrm{ml}$ ammonium persulfate $\left(\left(\mathrm{NH}_{4}\right)_{2} \mathrm{~S}_{2} \mathrm{O}_{8}, 1 \mathrm{M}\right)$, and $9 \mathrm{ml}$ of DDW. The formation of $\mathrm{Cu}(\mathrm{OH})_{2}$ nanowires [47, 48] and $\mathrm{Zn}(\mathrm{OH})_{2}$ nanostrands [49] are obtained after $30 \mathrm{~min}$ and $60 \mathrm{~min}$ of oxidation, respectively. The transformation of the metallic substrate into $\mathrm{Cu}(\mathrm{OH})_{2}$ nanowires or $\mathrm{Zn}(\mathrm{OH})_{2}$ nanostrands was confirmed using SEM and XRD.

\subsection{Preparation of precursor solutions and synthesis of MOFs films by IPL}


For the preparation of Cu-BTC films, BTC $(0.1 \mathrm{mM}, 0.5 \mathrm{mM}, 1 \mathrm{mM})$ are dissolved in $5 \mathrm{ml}$ of DMF. For the preparation of $\mathrm{Cu}-\mathrm{BDC}$ and MOF-5 films, the precursor solution is prepared by dissolving BDC (0.5 $\mathrm{mM}, 1 \mathrm{mM}, 3 \mathrm{mM})$ in $11 \mathrm{ml}$ of DMF. For the preparation of ZIF-8 films, 2-MIM (0.05 mM, $0.1 \mathrm{mM}, 0.5$ $\mathrm{mM}$ ) are dissolved in $100 \mathrm{ml}$ of DDW, and $10.1 \mathrm{ml}$ of TEA as a deprotonating agent. After stirring the precursor solutions vigorously for about $10 \mathrm{~min}$, approximately $100 \mu \mathrm{l}$ of the solution is drop-casted to cover the corresponding metal hydroxide surface, allowed to dry at room temperature (repeat three times) then subject to multiple IPL from a xenon flash light with an adjustable energy range from 3 to $27 \mathrm{~J}$ for 6 ms pulse. After the fabrication of MOFs films, the substrates are washed with copious amounts of DDW, ethanol and acetone, then dried by blowing nitrogen. The bare metal substrates are also checked for in-situ MOFs growth. However, the reaction does not happen due to the difficulty in the liberation of metal ions from bare metal substrates. The metal hydroxide layers facilitate liberation of metal ions during the IPL process. The overall process for the two-step MOFs films synthesis is described in Figure 1.

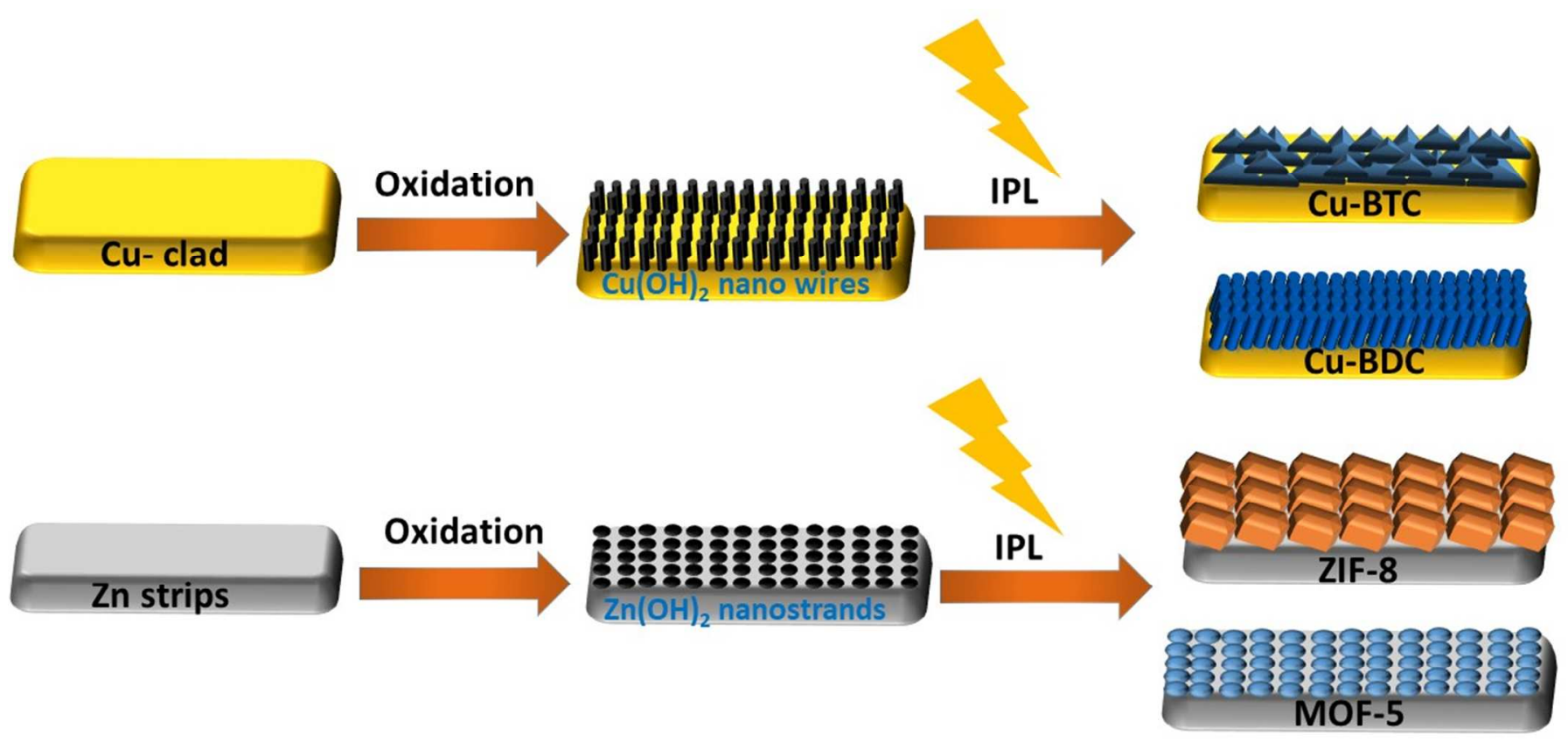

Fig. 1. Schematic illustration of the two-step method for MOFs films fabrication by IPL method. 


\subsection{Characterizations}

A field emission scanning electron microscope (FE-SEM, QuantaTM 250) is used to determine and analyze the morphologies and an X-ray diffractometer (XRD, Rigaku Multiflex) is used to characterize the crystalline structure of the two metal hydroxides and four MOFs films. UV-Visible spectrophotometer (Ocean Optics, Flame-S, USA) is used to measure the emission spectrum of xenon lamp and the reflectance spectra of the substrates before and after IPL-based MOFs synthesis. Adhesion tests of four MOFs films are performed by sonication method [25].

\section{Results and discussion}

MOFs films synthesis could be achieved by different methods such as LBL, LPE, LB, and electrochemical depositions [37, 40, 50]. However, film preparations may require longer time and the bonding between the substrate and MOFs films may be weak. Thus, a pretreatment of surface may be required through functionalizing the surface to attain good attachments [32]. Utilizing the metal substrate as a metal ion source offers a good choice to minimize the time of preparation and also enhance the attachment between the substrate and the organic ligands as in-situ MOFs film growth occurs. Thus, MOFs films can be prepared in good uniformity and homogeneity. In this work, the copper and zinc-based MOFs films are synthesized directly on copper and zinc substrates, respectively. Firstly, the metal substrates are oxidized to yield metal hydroxide layers. This step is important because transformation from metal to metal hydroxide facilitates the liberation of metal ions, consequently metal ions will coordinate with organic ligands to form the corresponding MOFs [51]. $\mathrm{Cu}$ and $\mathrm{Zn}$ metal substrates are treated with oxidizing solution to form $\mathrm{Cu}(\mathrm{OH})_{2}$ nanowires and $\mathrm{Zn}(\mathrm{OH})_{2}$ nanostrands as shown in Figure 2. The advantages of using metal hydroxide compared to bare metal substrate are coming from less binding energy and larger surface area. The $\mathrm{Cu}$ ion in hydroxide has binding energy of $916.5 \mathrm{eV}$ and $\mathrm{Cu}$ ion in metal has $932.61 \mathrm{eV}$. In addition, 
the $\mathrm{Zn}$ ion in hydroxide has binding energy of $1021 \mathrm{eV}$ and $\mathrm{Zn}$ ion in metal has $1021.65 \mathrm{eV}$ [52]. Also, the high surface area as shown in Figure 2(b) and (d) enables more chemical reactions compared to smooth surface as shown in Figure 2(a) and (c).

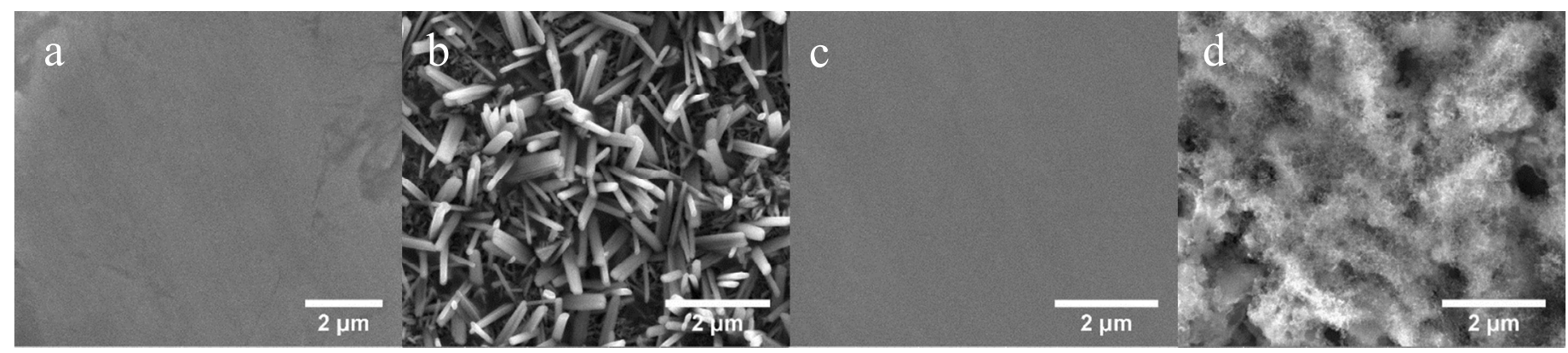

Fig. 2. SEM images of (a) $\mathrm{Cu}-\mathrm{clad}$, (b) $\mathrm{Cu}(\mathrm{OH})_{2}$ nanowires, (c) $\mathrm{Zn}$ strip, (d) $\mathrm{Zn}(\mathrm{OH})_{2}$ nanostrands.

$\mathrm{XRD}$ is used to confirm the conversion from metal $(\mathrm{Cu}$ or $\mathrm{Zn})$ to the corresponding metal hydroxides.

Figure 3(a) and (b) reveal that new peaks appear after oxidation of the metal substrate which represents conversion of pure metal to corresponding metal hydroxide. In the case of $\mathrm{Cu}$ shown in Figure 3(a), three new peaks at $2 \theta=16.9^{\circ}, 24.02^{\circ}$ and $34.28^{\circ}$ appear after oxidation which correspond to (202), (021) and (002), respectively, for $\mathrm{Cu}(\mathrm{OH})_{2}$ nanowires [53]. Four new peaks also appear for the $\mathrm{Zn}$ metal substrate as shown in Figure $3(\mathrm{~b})$ at $2 \theta=21.6^{\circ}, 32.38^{\circ}, 35^{\circ}$ and $48.1^{\circ}$ which correspond to (101), (100), (002) and (102), respectively, for $\mathrm{Zn}(\mathrm{OH})_{2}$ nanostrands [49, 54]. 

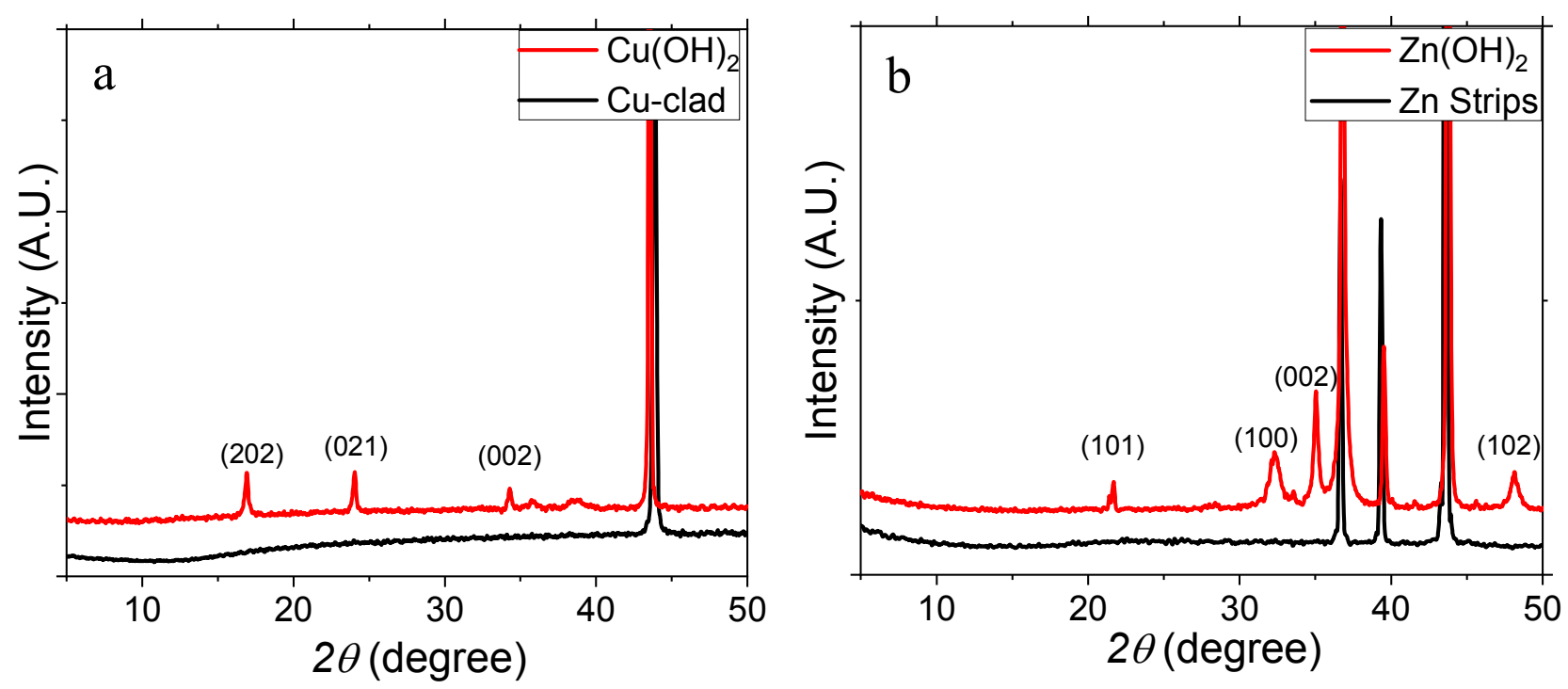

Fig. 3. XRD patterns of (a) $\mathrm{Cu}$-clad (black) and $\mathrm{Cu}(\mathrm{OH})_{2}$ nanowires on $\mathrm{Cu}$-clad substrate (red) and (b) $\mathrm{Zn}$ strip (black) and $\mathrm{Zn}(\mathrm{OH})_{2}$ nanostrands on $\mathrm{Zn}$ strip (red).

The organic ligand solutions are then used to convert $\mathrm{Cu}(\mathrm{OH})_{2}$ nanowires or $\mathrm{Zn}(\mathrm{OH})_{2}$ nanostrands to corresponding MOFs structure by subjecting to IPL. During the conversion process, the metal hydroxide nanowires or nanostrands are decorated by the organic ligand as an intermediate step for MOFs films formation. However, without IPL irradiation, amorphous layers of organic ligands over the metal hydroxide surfaces are just observed which implies that no proper reaction occurs between the organic ligand and the metal hydroxide for MOFs film formation (see supplementary section S1 Figure S1). Moreover, overall decreases of the hydroxide peaks are observed by XRD after drop and dry the organic ligand solutions without subjecting to any IPL pulses (see supplementary section S1 Figure S2). These are attributed to the formation of an amorphous layer on the top of the hydroxide layer. In addition, any peaks related to corresponding MOFs film formation are not observed.

The IPL exposure time is a crucial factor, in which each pulse takes approximately 6 milliseconds and 15 seconds of recharging. In this work, the MOFs films are not fully formed with less than 15 pulses. 
Exposing the oxidized substrate to 15 pulses which approximately takes 3 min makes a full conversion of metal hydroxide to MOFs films. In addition, exposure of more than 15 pulses has no observable effect on the density or homogeneity of four MOFs films (see supplementary section S2).

\subsection{UV-Vis measurements.}

IPL has the major contribution in our preparation strategy because it works as an energy source for the chemical reaction between metal ions and organic ligands. Therefore, the emission spectrum of the flashlight is first obtained and reflectance spectra of metal, metal hydroxide, MOFs films are investigated. Figure 4(a) shows the emission spectrum of IPL from a xenon lamp which has a peak emission wavelength around $580 \mathrm{~nm}$. Under the IPL, metal, metal hydroxide, and MOFs films can absorb photons with certain wavelengths depending on their absorption capacity. Subsequently, the temperature of the subjected area increases depending on the amount of absorbed photons with certain wavelengths. The reflectance of bare metal, metal hydroxide and MOFs films are obtained to investigate the absorption of photons from the xenon lamp. As shown in Figure 4(b) and (c), the reflectance spectra of $\mathrm{Cu}$-clad and $\mathrm{Zn}$ strips ranging from $400 \mathrm{~nm}$ to $800 \mathrm{~nm}$ are dramatically reduced after the oxidation step. $\mathrm{Cu}(\mathrm{OH})_{2}$ nanowires layer absorbs most of photons in this wavelength range and $\mathrm{Zn}(\mathrm{OH})_{2}$ nanostrands layer also facilitates absorption of more photons. Zn-based MOFs films further facilitate absorption of photons ranging from $400 \mathrm{~nm}$ to 800 nm. In addition, optical microscope images in Figure 4(b) and (c) show color change of each film investigated in this study which confirms reflectance spectrum acquired. Thus, localized and controlled photon energy is expected to be delivered into the two-step MOFs film synthesis process using IPL. 

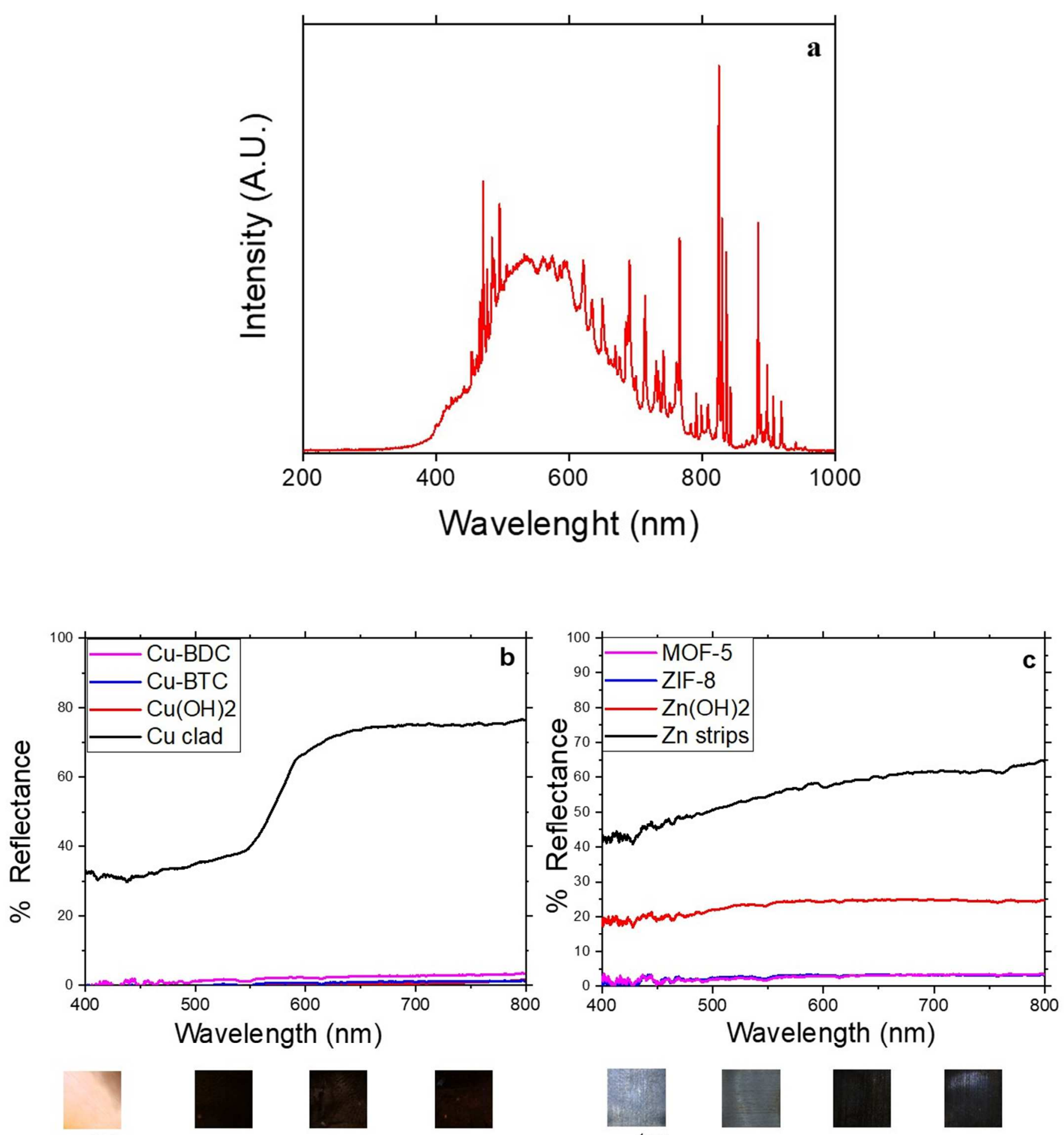

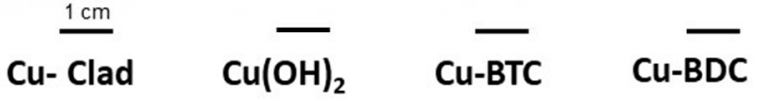

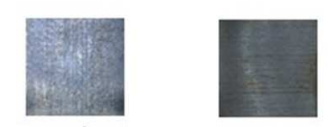

$1 \mathrm{~cm}$

Zn strips $\quad \mathrm{Zn}(\mathrm{OH})_{2} \quad$ ZIF-8
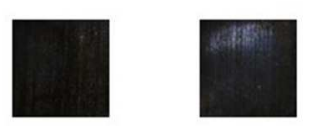

MOF-5

Fig. 4. (a) UV-Vis spectrum of the IPL from the xenon lamp and UV-Vis reflectance spectra of (b) Cubased and (c) Zn-based films coupled with optical microscope images of each film, respectively. 


\subsection{Synthesis of copper-based MOFs films: $\mathrm{Cu}-\mathrm{BTC}$ and $\mathrm{Cu}-\mathrm{BDC}$}

The primary mechanism in IPL method is localized and controlled heating of target materials. The multiple short exposures of photons with high energy generate highly transient temperature changes which promote chemical reactions between metal ions and organic ligands. Therefore, IPL exposure time is a crucial factor. The effects of varying numbers of IPL are systematically studied using SEM and XRD. The growth of MOFs films greatly depends on number of pulses and the dissolution rate of copper hydroxide. The protons from either BTC or BDC react with the copper hydroxide nanowires and generate copper ions on surface, which directly coordinate with BTC or BDC to form the corresponding MOFs. As shown in Figure 5(a) and (d), almost no $\mathrm{Cu}-\mathrm{BTC}$ or $\mathrm{Cu}-\mathrm{BDC}$ films have been formed with 5 pulses. Each film surface seems to be amorphous though some decorations of the copper hydroxide nanowires with corresponding organic ligands are observed. With 10 pulses, some $\mathrm{Cu}-\mathrm{BTC}$ and $\mathrm{Cu}-\mathrm{BDC}$ crystals are noticed in Figure 5(b) and (e) due to increasing number of pulses that most probably initiates a chemical reaction between copper ions $\left(\mathrm{Cu}^{+2}\right)$ and organic ligands. With 15 pulses, homogeneous films of $\mathrm{Cu}-\mathrm{BTC}$ and $\mathrm{Cu}-\mathrm{BDC}$ are easily recognized as shown in Figure 5(c) and (f), respectively. It seems that sufficient energy is delivered to the reaction medium, consequently dense and homogeneous MOFs films are fully formed. Exposing the oxidized substrate to 15 pulses which approximately takes 3 min makes a full conversion of metal hydroxide to MOFs film. In addition, exposure of more than 15 pulses has no observable effect on the film density or homogeneity (see supplementary section S2 Figure S3). We notice that Cu-BTC or Cu-BDC crystal size is relatively smaller compared to the size usually found in the literature $[5,12,41,55]$. This smaller size of the $\mathrm{Cu}-\mathrm{BTC}$ or $\mathrm{Cu}-\mathrm{BDC}$ crystals is attributed to shorter reaction time in addition to highly transient reaction temperature [56]. 

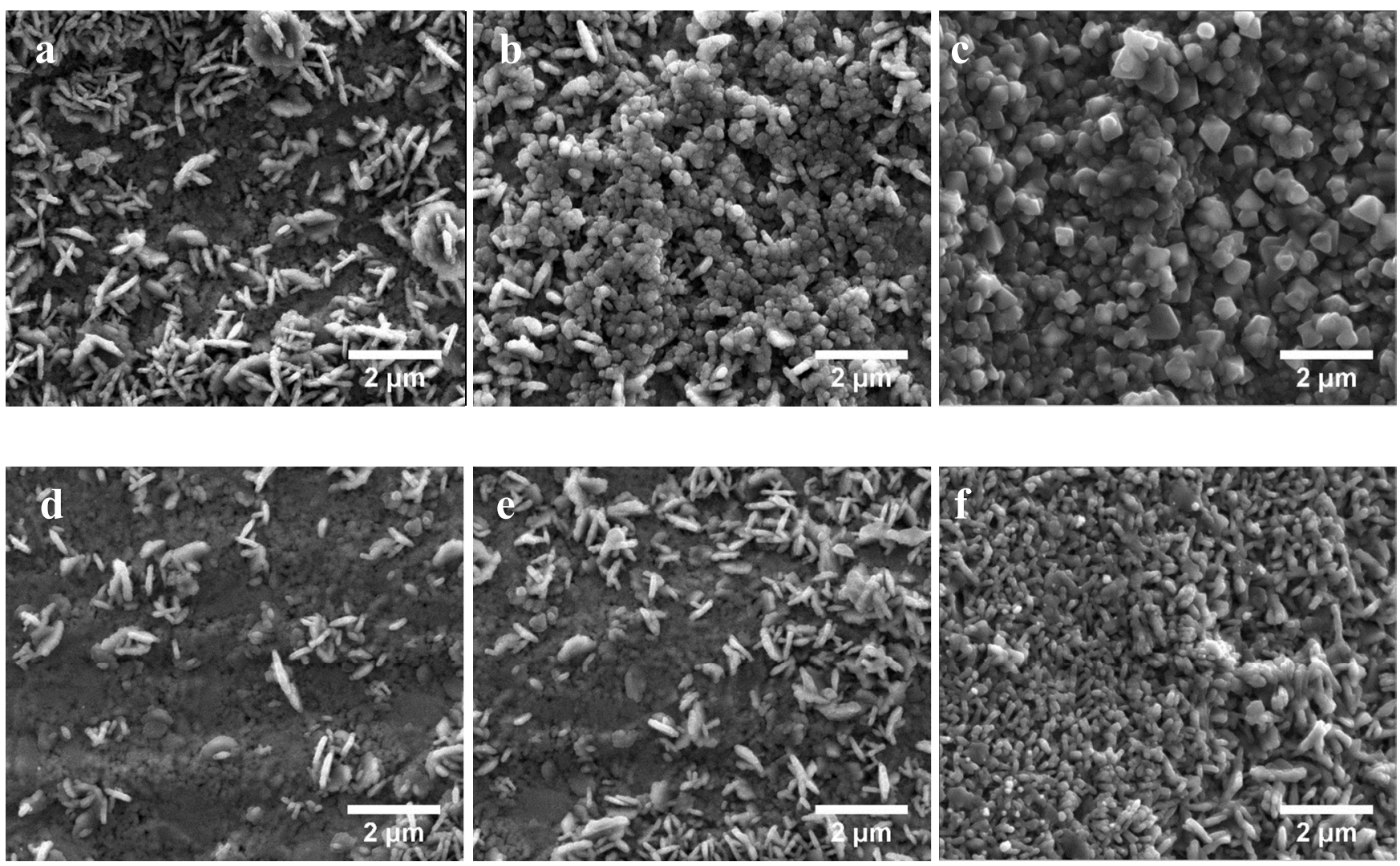

Fig. 5. SEM images of Cu-BTC with $0.5 \mathrm{mM} \mathrm{BTC}$ as a function of varying number of IPL, (a) Cu-BTC with 5 pulses, (b) Cu-BTC with 10 pulses, (c) Cu-BTC with 15 pulses. The second row shows SEM images of $\mathrm{Cu}-\mathrm{BDC}$ with $1 \mathrm{mM}$ BDC as a function of varying number of IPL, (d) Cu-BDC with 5 pulses, (e) CuBDC with 10 pulses, (f) Cu-BDC with 15 pulses.

Figure 6(a) shows the XRD patterns of Cu-BTC film with $0.5 \mathrm{mM}$ BTC as a function of varying number of IPL. With 5 pulses, no peaks related to $\mathrm{Cu}-\mathrm{BTC}$ are detected. Moreover, no peaks of $\mathrm{Cu}(\mathrm{OH})_{2}$ nanowires are detected. This may be due to the decoration of $\mathrm{Cu}(\mathrm{OH})_{2}$ with organic ligands and insufficient energy delivered to the reaction medium to form $\mathrm{Cu}-\mathrm{BTC}$ crystals. With 10 pulses, small distinct peaks appear at $2 \theta=6.82^{\circ}, 9.64^{\circ}$ and $11.74^{\circ}$, which correspond to (200), (220) and (222), respectively [57]. The intensity of these peaks increases with 15 pulses in which the crystals become more uniform and fully converted to 
$\mathrm{Cu}$-BTC film structure. The strongest XRD peak at $2 \theta=11.74^{\circ}$ which corresponds to the crystallographic plane (222) direction suggests the epitaxial growth of the Cu-BTC film. Thus, the role of the metal substrate and IPL are not only to act as nucleation template for the film growth, but also to control the growth direction.

Figure 6(b) shows the XRD patterns of $\mathrm{Cu}-\mathrm{BDC}$ film with $1 \mathrm{mM} \mathrm{BDC}$ as a function of varying number of IPL. With 5 pulses, no peaks related to $\mathrm{Cu}-\mathrm{BDC}$ are detected, which may suggest that no chemical reaction happens. With 10 pulses, one peak appears at $2 \theta=17.6^{\circ}$ which corresponds to (002) of $\mathrm{Cu}-\mathrm{BDC}$ that suggests formation of some $\mathrm{Cu}-\mathrm{BDC}$ rod crystal structure. With 15 pulses, homogenous $\mathrm{Cu}-\mathrm{BDC}$ film is attained where the rod structure of $\mathrm{Cu}-\mathrm{BDC}$ is noticed everywhere on the film surface. In addition, distinct peaks appear at $2 \theta=7.8^{\circ}$ and $17.6^{\circ}$ which correspond to (001) and (002) of $\mathrm{Cu}-\mathrm{BDC}$, respectively [1]. The rod structure of $\mathrm{Cu}-\mathrm{BDC}$ proceeds epitaxially along (001) direction.
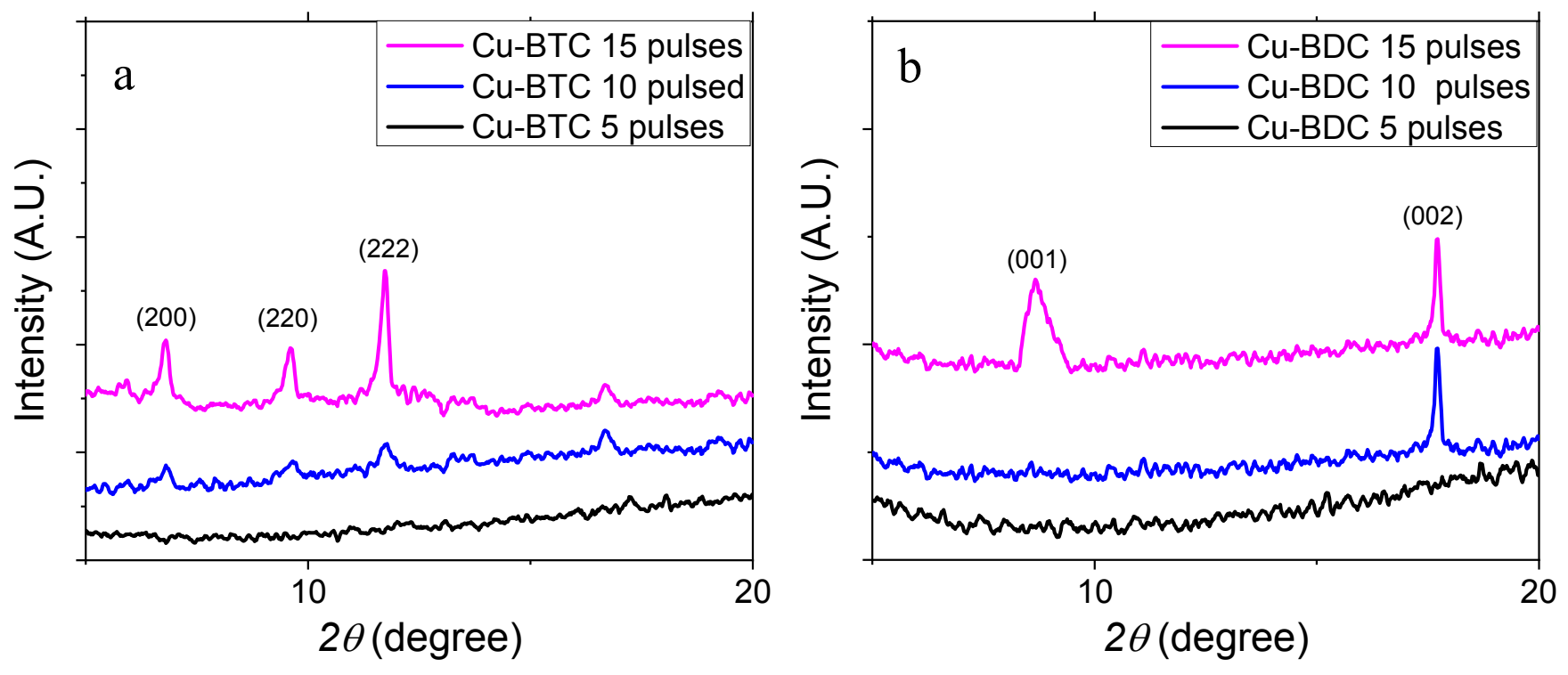

Fig. 6. XRD patterns of (a) Cu-BTC films with $0.5 \mathrm{mM} \mathrm{BTC}$, and (b) $\mathrm{Cu}-\mathrm{BDC}$ films with $1 \mathrm{mM}$ BDC as a function of varying number of IPL. 


\subsection{Synthesis of zinc-based MOFs films: ZIF-8 and MOF-5}

ZIF- 8 crystals, made up of $\mathrm{Zn}^{2+}$ ions and imidazole organic ligands, are outstandingly different MOFs from $\mathrm{Cu}-\mathrm{BDC}, \mathrm{Cu}-\mathrm{BTC}$ and MOF-5. The latters are MOFs bounded via oxygen coordination, whereas ZIF-8 is a MOFs bounded via nitrogen coordination. The proposed mechanism of the reaction is similar to Cu-based MOFs discussed in Section 3.2. The protons from TEA and BDC react with the $\mathrm{Zn}(\mathrm{OH})_{2}$ to generate $\mathrm{Zn}^{+2}$ ions that coordinate with 2-MIM and BDC to form ZIF-8 and MOF-5 crystals, respectively. Figure 7(a) to (c) show the growth of ZIF-8 crystals as a function of varying number of IPL. The crystals are moderately cubic and have rough surfaces but some angles can be observed on some of them. They are varied from cubes exposing 6 faces to intermediate shapes, and finally to parallelogram exposing 12 faces, usually the latter shape can be considered the most stable equilibrium morphology of ZIF-8. As shown in Figure 7(c), good crystalline shapes with uniform morphology appear with 15 pulses. Less than 15 pulses, some crystals are formed but they are not developed well because no sufficient energy is delivered to facilitate the reaction between zinc ions $\left(\mathrm{Zn}^{+2}\right)$ and organic ligands. For the synthesis of MOF-5, Figure 7(d) to (f) show the crystal morphology of MOF-5 film after subjecting to varying number of IPL. As visualized by SEM, smooth, monodisperse, spherical MOF-5 crystals with average diameter of $58 \mathrm{~nm}$ (calculated crystal size by Scherrer equation with XRD pattern in Figure 8(b) is $52 \mathrm{~nm}$ ) are produced with 15 pulses. With less number of pulses (5 and 10), some crystals are observed, however, the crystals seem to be aggregated without defined edges as noticed in Figure 7(d) and (e).
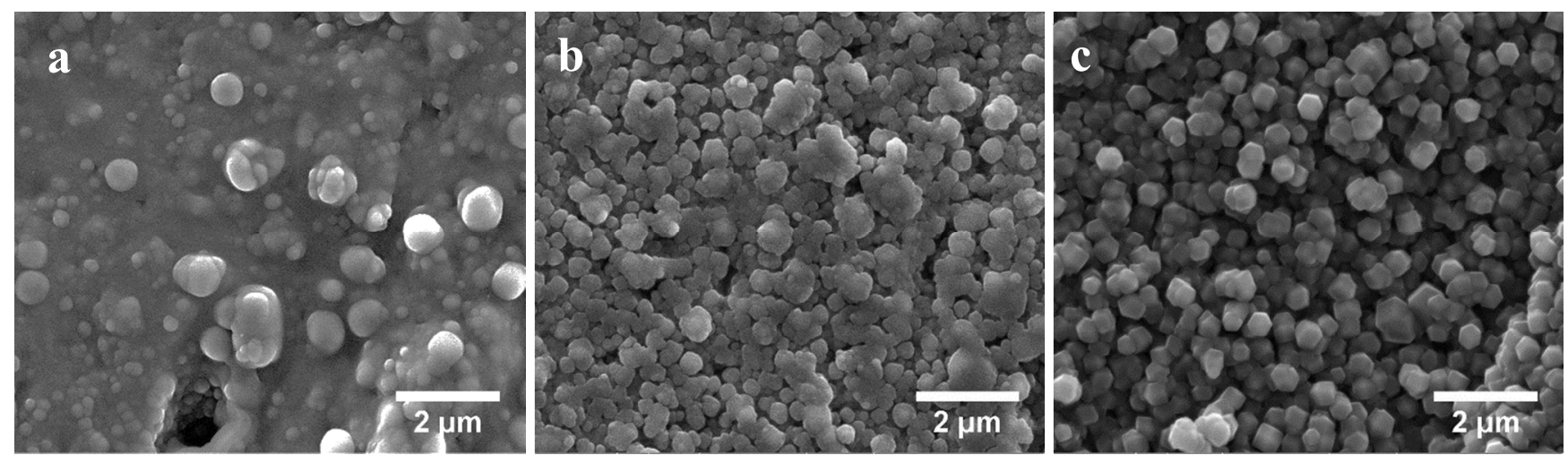


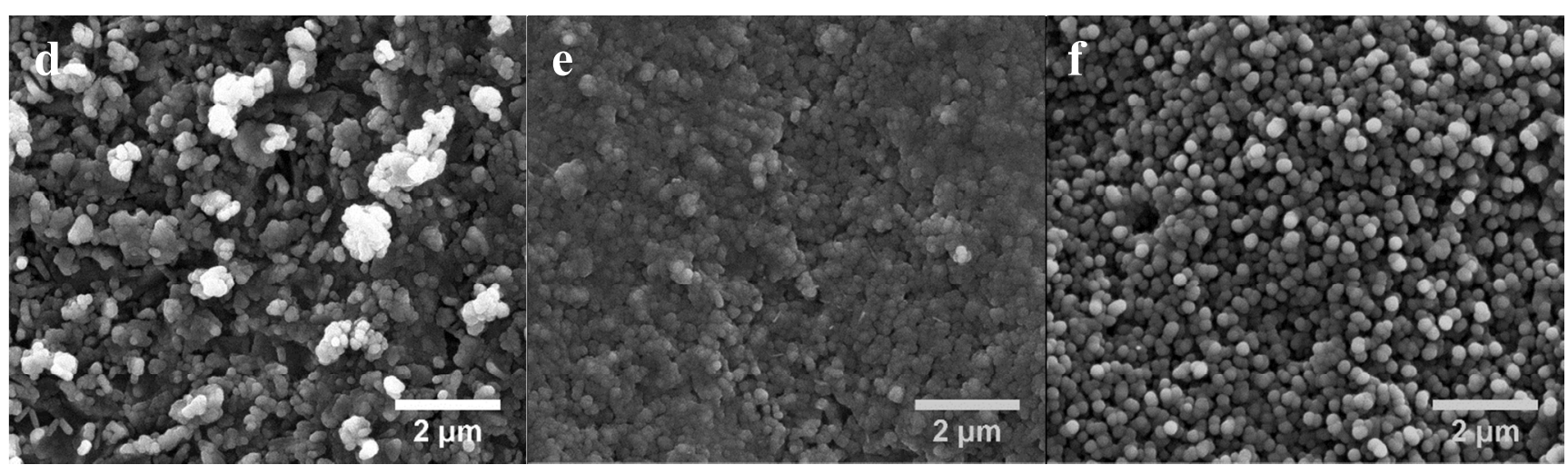

Fig. 7. SEM images of ZIF-8 with $0.1 \mathrm{mM}$ 2-MIM as a function of varying number of pulses, (a) ZIF-8

with 5 pulses, (b) ZIF-8 with 10 pulses, (c) ZIF-8 with 15 pulses. The second row shows SEM images of MOF-5 with $1 \mathrm{mM} \mathrm{BDC}$ as a function of varying number of pulses, (d) MOF-5 with 5 pulses, (e) MOF-5 with 10 pulses, (f) MOF-5 with 15 pulses.

Figure 8(a) shows the XRD patterns of the ZIF-8 films with $0.1 \mathrm{mM} 2-\mathrm{MIM}$ as a function of varying number of IPL. The XRD pattern produced by the well-organized porous structure of the ZIF-8 crystals is noticed with 15 pulses. The comparative intensities and notable peak positions at $2 \theta=7.7^{\circ}, 10.7^{\circ}, 13^{\circ}$, $18.5^{\circ}, 25^{\circ}$, and $27.1^{\circ}$ which correspond to (011), (002), (112), (222), (233) and (044), respectively, are in good agreement with previous reports [58-60]. This confirms the structure of ZIF-8 crystals and sharp peaks reveal high crystallinity of ZIF-8. With 10 pulses, tiny peaks related to the formation of some ZIF-8 crystals are observed. However, the crystallinity is not good enough to show more peaks related to ZIF-8. The most intense peak at $2 \theta=7.7^{\circ}$ that corresponds to (011) suggests the direction of ZIF- 8 crystal growth that is also epitaxially grown in that direction. The utilizing of metal substrate and IPL gives a preference for growth direction in the (011) direction.

Figure 8(b) shows the XRD patterns of the MOF-5 films with $1 \mathrm{mM}$ BDC as a function of varying number of IPL. The appearance of five peaks at $2 \theta=9.2^{\circ}, 14.8^{\circ}, 16.1^{\circ}, 17.8^{\circ}$ and $28.9^{\circ}$, which correspond to (220) , (400), (420), (440), (751), respectively, suggests the formation of a MOF-5 film is achieved [15, 42]. 
Some tiny peaks related to the formation of some crystals are observed with 5 and 10 pulses. However, the crystallinity is not good enough to show more peaks related to MOF-5. It could be concluded that both $\mathrm{Cu}$ and Zn-based MOFs are fully developed with good homogeneity and crystallinity with 15 pulses (approximately $3 \mathrm{~min}$ ). These conditions deliver the sufficient energy to grow $\mathrm{Cu}$ and $\mathrm{Zn}$-based MOFs films over the metal substrates.
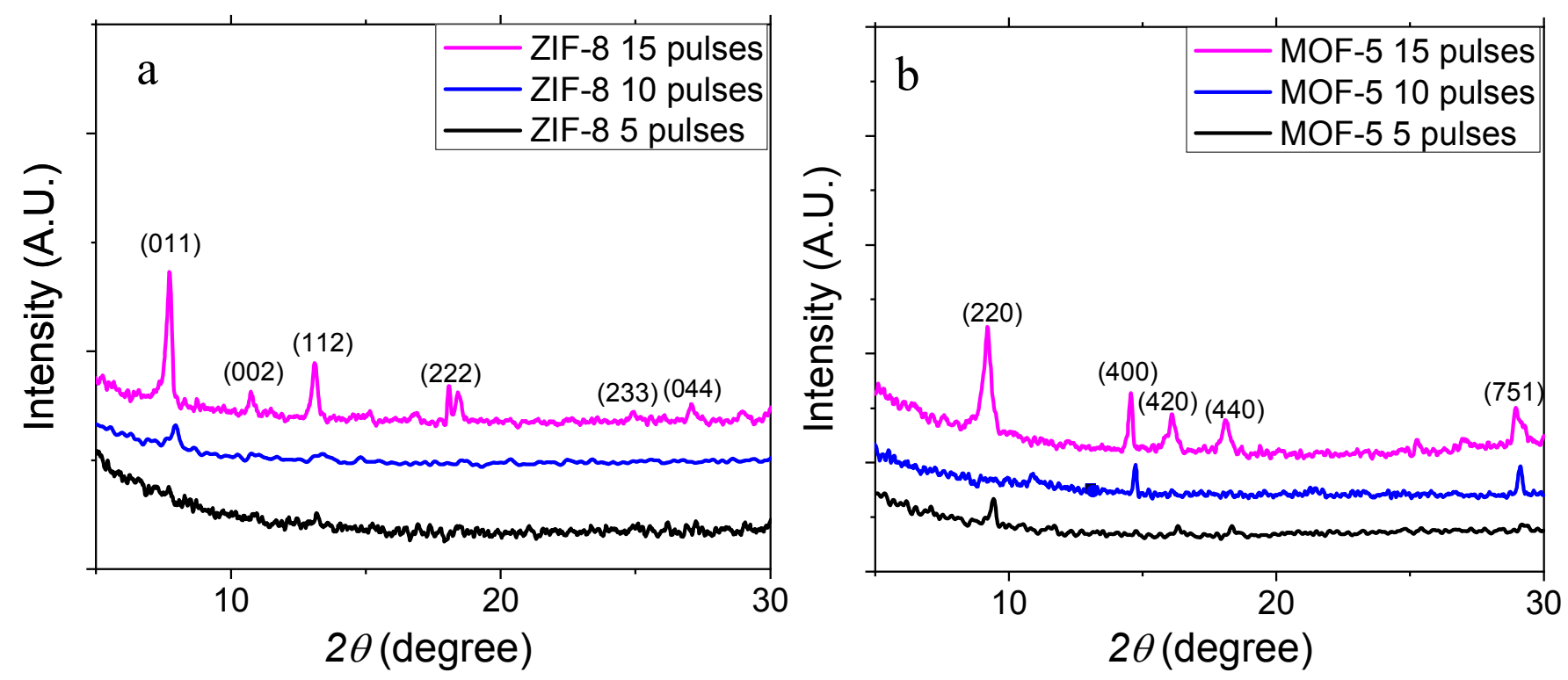

Fig. 8. XRD patterns of (a) ZIF-8 films with $0.1 \mathrm{mM}$ 2-MIM, and (b) MOF-5 films with $1 \mathrm{mM}$ BDC as a function of varying number of IPL.

\subsection{Effect of different concentration of organic ligands on MOFs films synthesis}

The morphology and crystallinity of MOFs films are greatly affected by the organic ligands concentration. Thus, the rapid and facile synthesis of the four MOFs films is systematically studied as a function of organic ligands concentration. All MOFs films used for studying the effect of organic ligands concentration are prepared with 15 pulses of IPL. Figure 9 and 10 demonstrate the morphology and crystallinity of the four MOFs films as a function of organic ligands concentration. At $0.1 \mathrm{mM}$ of BTC organic ligands 
concentration, some Cu-BTC crystals are observed with SEM (see Figure 9(a)). However, the cubic shapes of $\mathrm{Cu}$-BTC crystals are not reached yet and the $\mathrm{Cu}$-BTC film is neither uniform nor dense. This may be due to the lack of organic ligands ions in this low concentration. Consequently, no relevant peaks of $\mathrm{Cu}-\mathrm{BTC}$ are detected with XRD as shown in Figure 10(a). In contrast, cubic structures of Cu-BTC crystals with well-defined edges are observed at $0.5 \mathrm{mM}$ and $1 \mathrm{mM}$ of BTC (see Figure 9(b) and (c)). This is evident with three XRD peaks related to $\mathrm{Cu}-\mathrm{BTC}$ (see Figure 10(a)). For the synthesis of $\mathrm{Cu}-\mathrm{BDC}$ film, very few rod structures are observed at $0.5 \mathrm{mM}$ of BDC (see Figure 9(d)). However, at $1 \mathrm{mM}$ and $3 \mathrm{mM}$ of BDC, rod structures of $\mathrm{Cu}$-BDC crystals are easily recognized with SEM images although the homogeneity and density seem to be better at $1 \mathrm{mM}$ of BDC (see Figure 9(e) and (f)). As shown in Figure 10(b), no peaks for $\mathrm{Cu}-\mathrm{BDC}$ are recognized at $0.5 \mathrm{mM}$ of BDC. However, at $1 \mathrm{mM}$ and $3 \mathrm{mM}$ of BDC, two peaks correspond to $\mathrm{Cu}-\mathrm{BDC}$ are observed which confirm the formation of $\mathrm{Cu}-\mathrm{BDC}$ crystals.

ZIF-8 film is synthesized at $0.05 \mathrm{mM}$ of 2-MIM as shown in Figure $9(\mathrm{~g})$. Crystals of ZIF-8 with noticeable sharp edges are achieved in this low concentration. This is also reflected to XRD pattern in Figure 10(c), multiple peaks of ZIF-8 films are detected even at this low concentration. The size of crystals is smaller than that of crystals formed at $0.1 \mathrm{mM}$ of 2-MIM (see Figure 9(h)). However, at $0.5 \mathrm{mM}$ of 2-MIM, big crystals are observed which may be formed due to aggregation of ZIF-8 particles (see supplementary section S3 Figure S4(a)). As shown in Figure 10(c), the XRD peak intensities increase due to the formation of big crystals at this high concentration ( $0.5 \mathrm{mM}$ of 2-MIM). Moreover, new peaks appear at $2 \theta=14.9^{\circ}$, $16.66^{\circ}, 20.46^{\circ}, 21.12^{\circ}, 22.34^{\circ}$ and $24.7^{\circ}$. These new peaks are most probably due to formation of very big crystals which also has different crystal shape (octahedral) compared to ZIF-8 crystal (cubic). The base film is also composed of ZIF-8 crystals but the shape is not developed well (see supplementary section S3 Figure S4(a)). 
In case of MOF-5, the morphology of the film is good at $1 \mathrm{mM}$ of BDC (see Figure 9(k)). At lower concentration ( $0.5 \mathrm{mM}$ of BDC), small crystals of MOF-5 are observed although the crystal growth seems to be incomplete due to the lack of organic ligands ions (see Figure 9(j)). Interestingly, no relevant XRD peaks are detected at this low concentration $(0.5 \mathrm{mM}$ of BDC) in Figure 10(d). At $3 \mathrm{mM}$ of BDC, big crystals with irregular shapes are distributed all over the surface (see supplementary section S3 Figure S4(b)). Aggregation of MOF-5 crystals seems to happen and residue of unreacted organic ligands seems to contribute to form these irregular crystal shapes. In addition, some new peaks appear at $2 \theta=12.55^{\circ}, 21^{\circ}$ and $25.5^{\circ}$ on XRD pattern which may correspond to the irregular crystals formed at this high concentration. In conclusion, organic ligands concentration has a great effect on MOFs synthesis process. Lower concentration may develop MOFs crystals with smaller sizes. In addition, the crystallinity and homogeneity of MOFs crystals may be affected due to the decrease in the nucleation and growth rates. A process optimization is highly recommended to synthesize well-developed MOFs crystals.
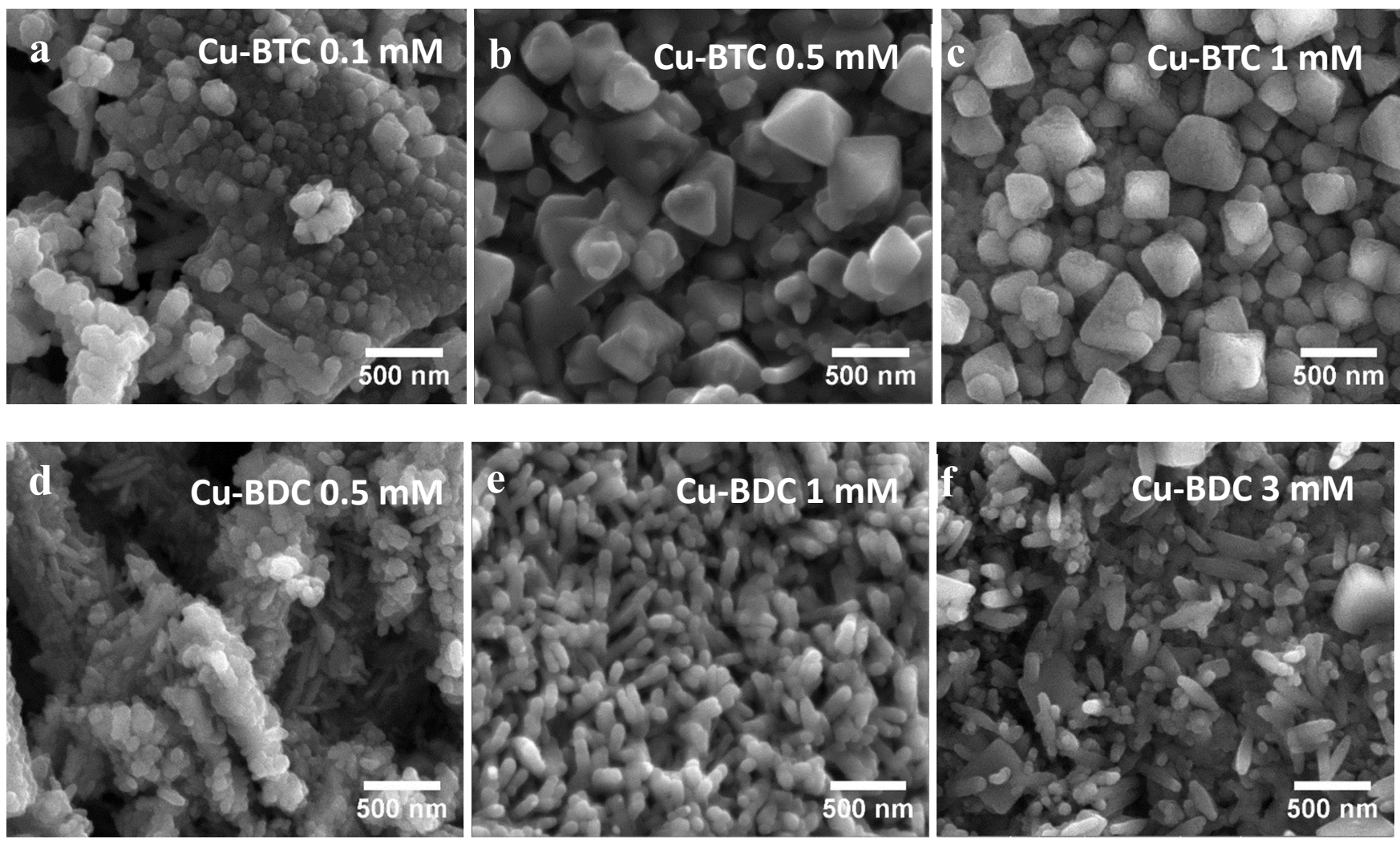

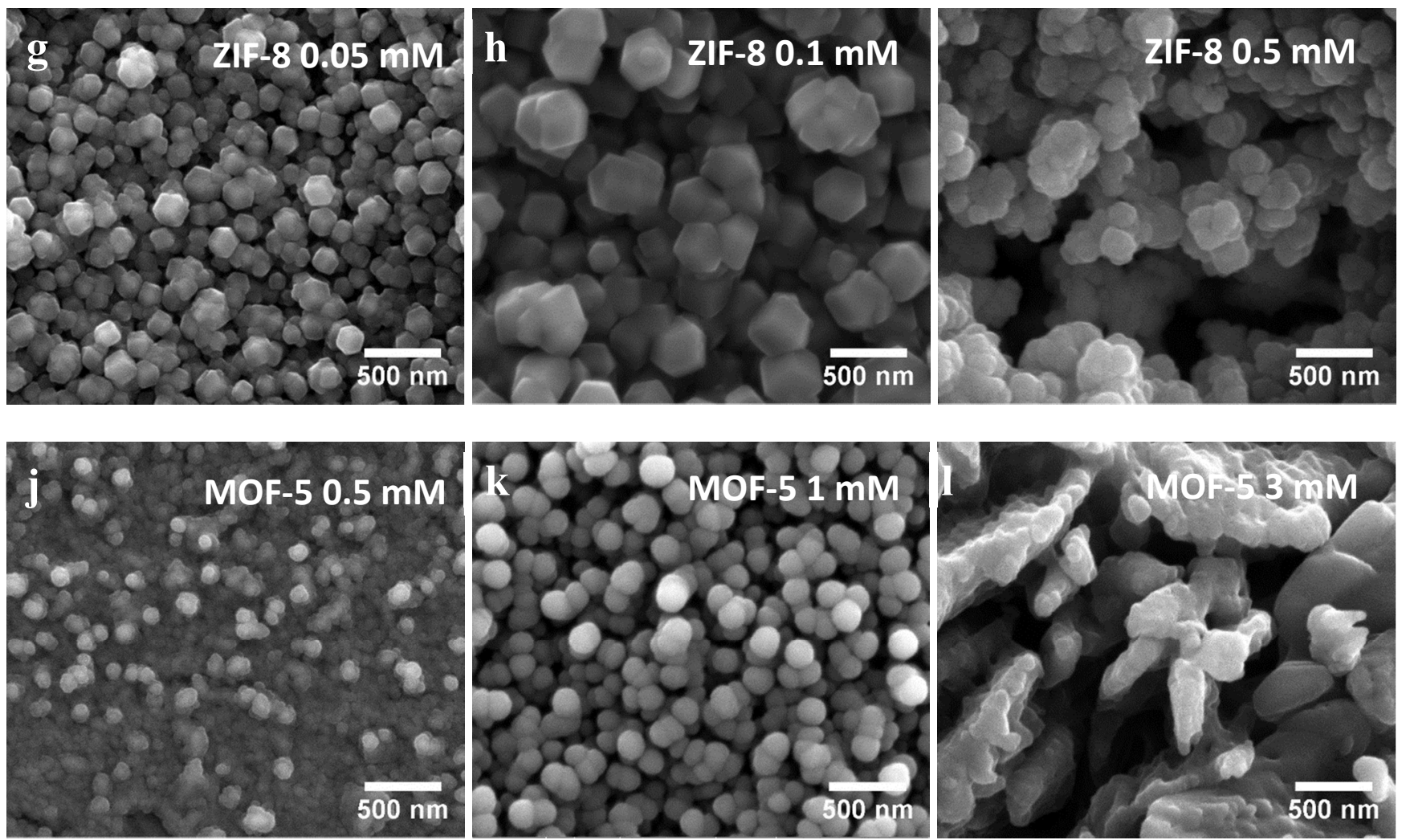

Fig. 9. SEM images of (a) Cu-BTC with $0.1 \mathrm{mM} \mathrm{BTC}$, (b) Cu-BTC with $0.5 \mathrm{mM} \mathrm{BTC}$, (c) Cu-BTC with 1

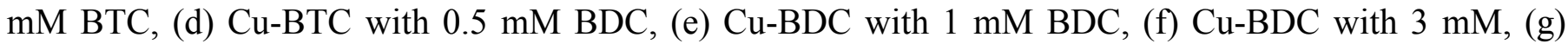
ZIF-8 with $0.05 \mathrm{mM}$ 2-MIM, (h) ZIF-8 with $0.1 \mathrm{mM}$ 2-MIM, (i) ZIF-8 with $0.5 \mathrm{mM}$ 2-MIM, (j) MOF-5 with $0.5 \mathrm{mM}$ BDC, (k) MOF-5 with $1 \mathrm{mM}$ BDC, and (l) MOF-5 with $3 \mathrm{mM} \mathrm{BDC}$.
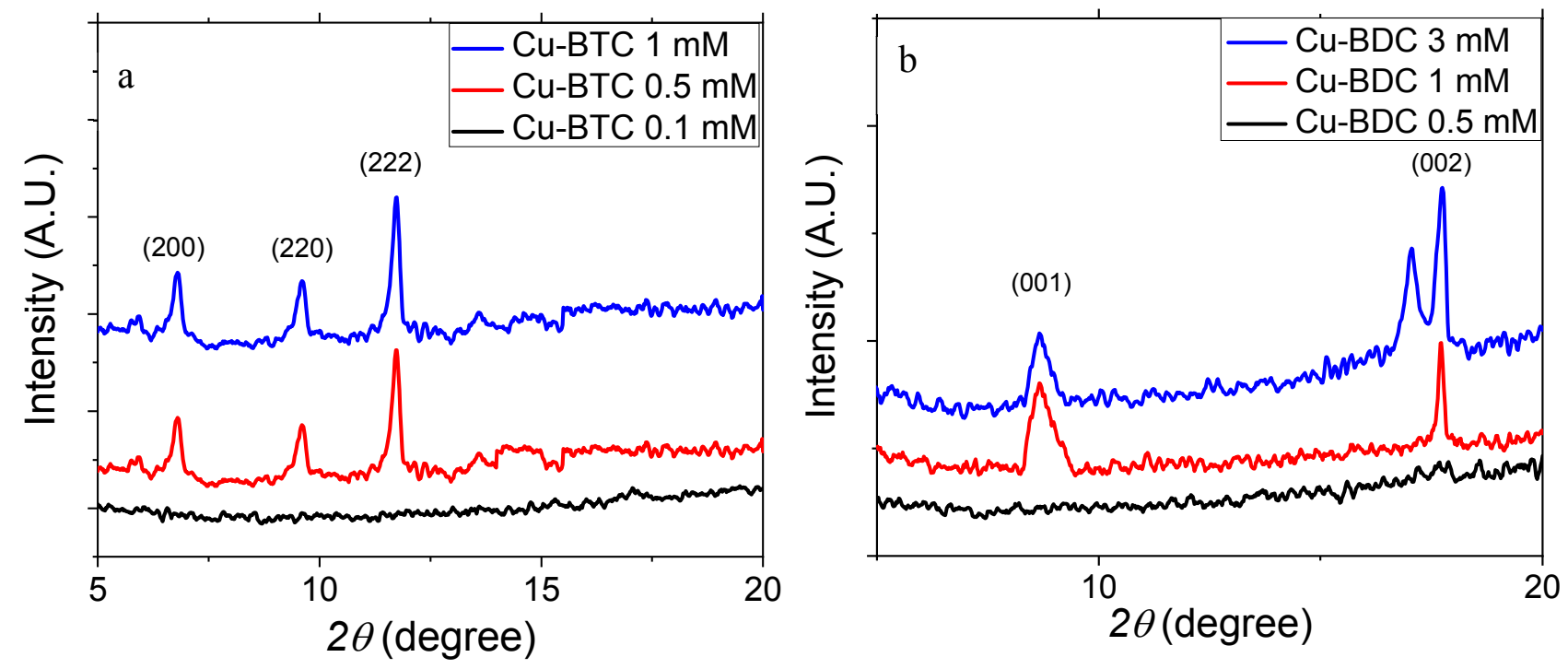

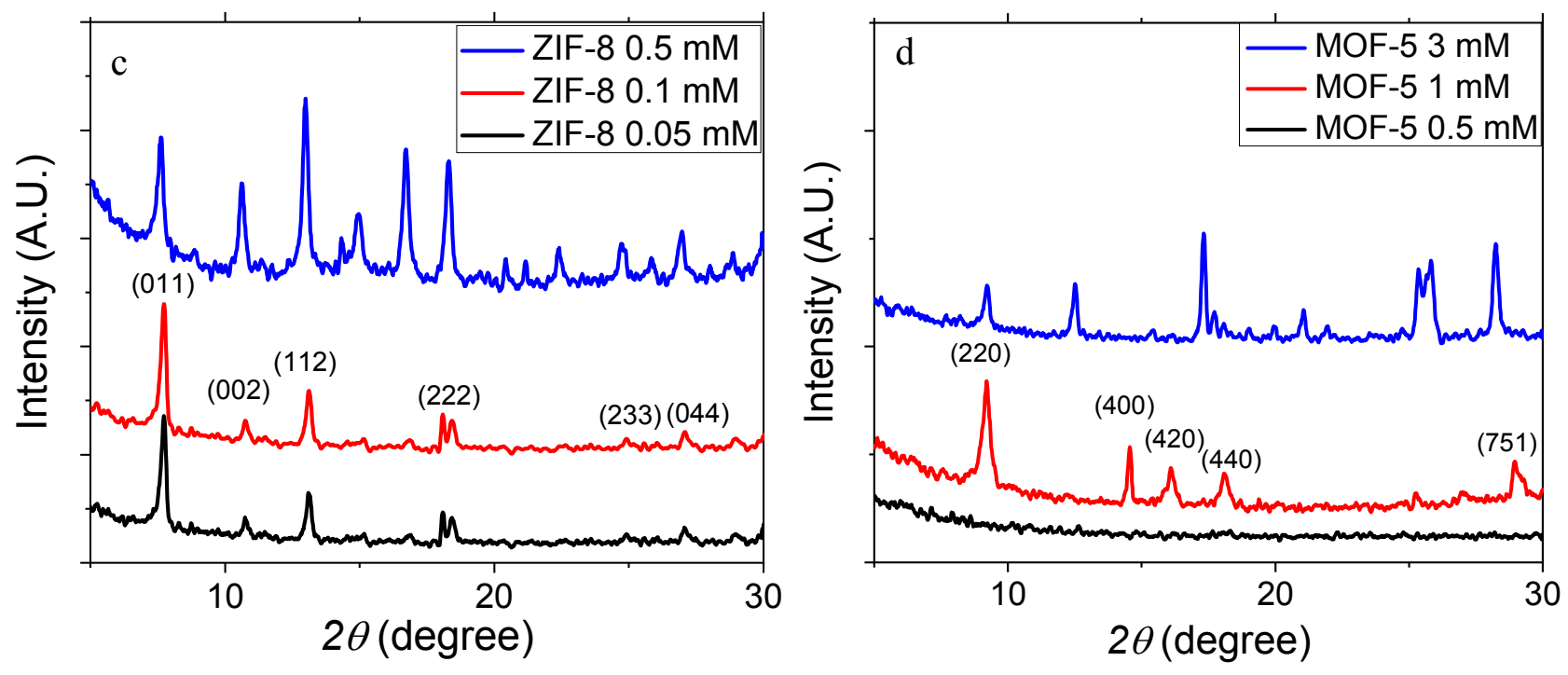

Fig. 10. XRD patterns of (a) Cu-BTC, (b) Cu-BDC, (c) ZIF-8, and (d) MOF-5 films at different organic ligand concentrations.

\subsection{Adhesion test of the MOFs films on metal substrate}

Strong adhesion of a MOF film at the interface between the film and the metal substrate is important especially in gas sensing applications. The adhesion of the four MOFs films prepared by IPL method are tested with sonication method [25]. Four MOFs films, Cu-BTC with $0.5 \mathrm{mM} \mathrm{BTC,} \mathrm{Cu-BDC}$ with $1 \mathrm{mM}$ BDC, ZIF-8 with $0.1 \mathrm{mM}$ 2-MIM, and MOF-5 with $1 \mathrm{mM} \mathrm{BDC}$, are prepared by IPL method then placed separately on vials containing ethanol. The vials are sonicated in an ultrasonic bath $(40 \mathrm{kHz}$ and ultrasonic power of $100 \mathrm{~W}$ ) for up to $60 \mathrm{~min}$. The attachment of each MOFs film at several intervals from 5 min up to 60 min is tested. SEM images in Figure 11 show that the MOFs films have good adhesion as most crystals remain still attached to the metal substrate after $60 \mathrm{~min}$ of sonication. Moreover, before and after ultrasonication test, side-view SEM images are taken to estimate the thicknesses of four MOFs films (see supplementary section S4 Figure S5). The film thickness is in the range of hundreds of nanometer to $1 \mu \mathrm{m}$. 


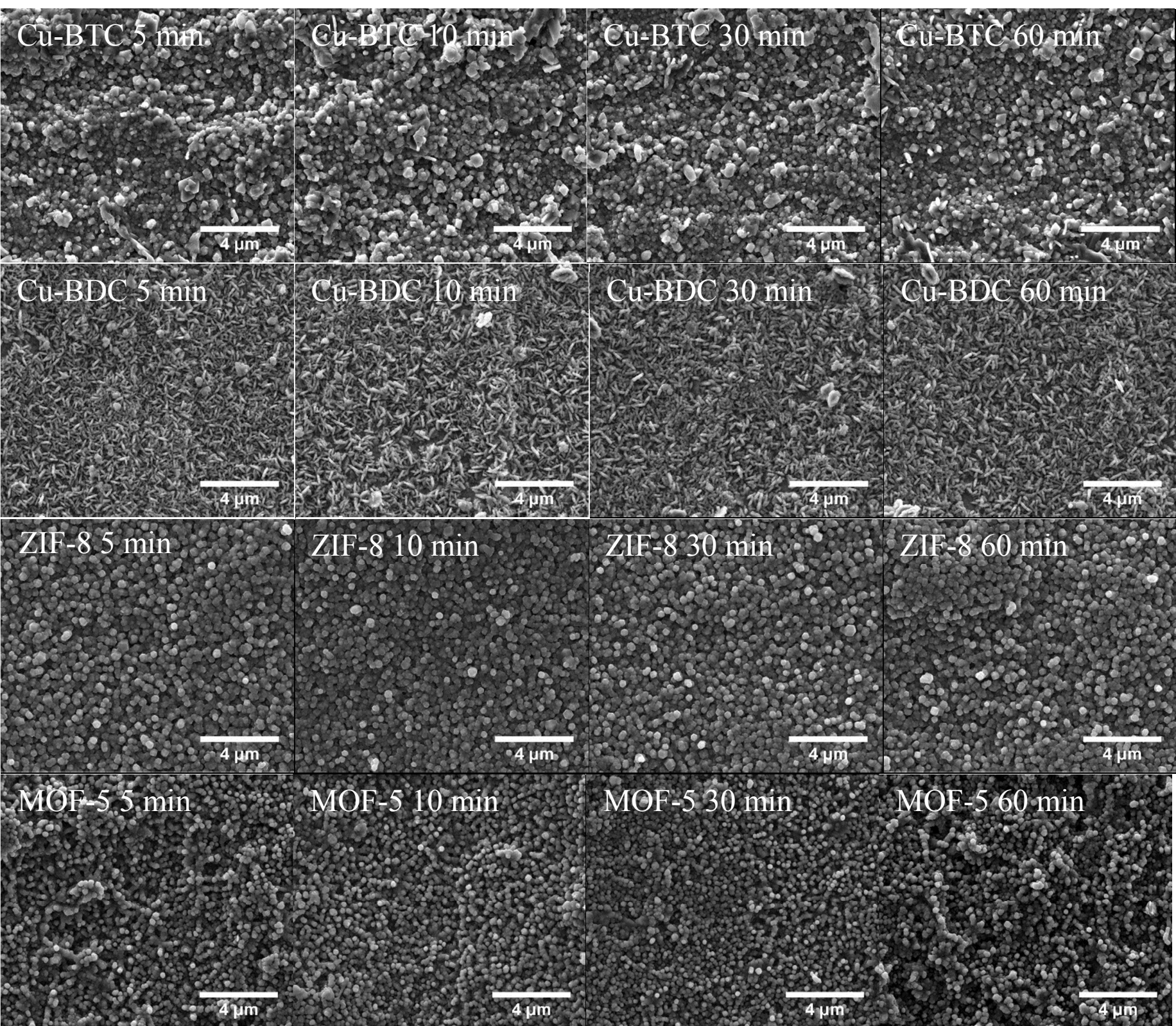

Fig. 11. SEM images of Cu-BTC, Cu-BDC, ZIF-8, and MOF-5 films taken after sonication for 5, 10, 30, and $60 \mathrm{~min}$, from left to right.

\section{Conclusions}

A facile and rapid approach is demonstrated for the two-step growth of four MOFs films (i.e., Cu-BTC, $\mathrm{Cu}-\mathrm{BDC}$, ZIF-8, and MOF-5) onto metal substrates with IPL. The process is carried out at ambient conditions using low-cost equipment. The procedure has the advantages of short processing time and 
versatility towards the preparation of different MOFs compounds. IPL has the major contribution in this approach because it works as an energy source required stimulating the chemical reaction between metal ion and organic ligands. The exposure time to IPL is a crucial factor. The MOFs films are fully formed with 15 pulses. The optimal concentrations of organic ligands are $0.5 \mathrm{mM} \mathrm{BTC}$ for $\mathrm{Cu}-\mathrm{BTC}$; $0.1 \mathrm{mM} 2$ MIM for ZIF-8; and $1 \mathrm{mM} \mathrm{BDC}$ for MOF-5 and Cu-BDC. The films are fully converted and homogeneous at these concentrations. This approach is expected to be useful in preparation of other MOFs, such as MOF-74, IRMOF-62 MIL-101, MOF-199 for different applications in gas sensing and gas separation.

\section{Acknowledgments}

The authors are very grateful for the support from the Natural Sciences and Engineering Research Council of Canada (NSERC) Discovery Grant Program. O.A and M.E. would like also acknowledge Military Technical College, Egyptian Armed Force for their financial support.

\section{References}

1. Yim, C. and S. Jeon, Direct synthesis of CU-BDC frameworks on a quartz crystal microresonator and their application to studies of n-hexane adsorption. RSC Advances, 2015. 5(83): p. 67454-67458.

2. Baumann, T., Metal-Organic Frameworks: Literature Survey and Recommendation of Potential Sorbent Materials. 2010, Lawrence Livermore National Laboratory (LLNL), Livermore, CA.

3. Britt, D., D. Tranchemontagne, and O.M. Yaghi, Metal-organic frameworks with high capacity and selectivity for harmful gases. Proceedings of the National Academy of Sciences, 2008. 105(33): p. 1162311627.

4. Abuzalat, O., et al., Sonochemical Fabrication of CU (II) and Zn (II) Metal-Organic Framework Films on Metal Substrates. Ultrasonics Sonochemistry, 2018.

5. MacGillivray, L.R., Metal-Organic Frameworks: Design and Application. 2010: Wiley.

6. Mueller, U., et al., Metal-organic frameworks-prospective industrial applications. Journal of Materials Chemistry, 2006. 16(7): p. 626-636.

7. Stock, N. and S. Biswas, Synthesis of metal-organic frameworks (MOFs): routes to various MOF topologies, morphologies, and composites. Chemical reviews, 2011. 112(2): p. 933-969.

8. Furukawa, H., et al., Ultrahigh porosity in metal-organic frameworks. Science, 2010. 329(5990): p. 424-428.

9. Küsgens, P., et al., Characterization of metal-organic frameworks by water adsorption. Microporous and Mesoporous Materials, 2009. 120(3): p. 325-330. 
10. Chen, B., et al., Zeolitic imidazolate framework materials: recent progress in synthesis and applications. Journal of Materials Chemistry A, 2014. 2(40): p. 16811-16831.

11. Zhou, H.-C., J.R. Long, and O.M. Yaghi, Introduction to metal-organic frameworks. 2012, ACS Publications.

12. MacGillivray, L.R. and C.M. Lukehart, Metal-Organic Framework Materials. 2014: Wiley.

13. Rowsell, J.L. and O.M. Yaghi, Metal-organic frameworks: a new class of porous materials. Microporous and Mesoporous Materials, 2004. 73(1): p. 3-14.

14. Shekhah, O., et al., Step-by-step route for the synthesis of metal-organic frameworks. Journal of the American Chemical Society, 2007. 129(49): p. 15118-15119.

15. Li, J., et al., Synthesis and hydrogen-storage behavior of metal-organic framework MOF-5. international journal of hydrogen energy, 2009. 34(3): p. 1377-1382.

16. Dey, C., et al., Crystalline metal-organic frameworks (MOFs): synthesis, structure and function. Acta Crystallographica Section B: Structural Science, Crystal Engineering and Materials, 2014. 70(1): p. 3-10.

17. Kaskel, S., The Chemistry of Metal-Organic Frameworks: Synthesis, Characterization, and Applications. 2016: Wiley.

18. Furukawa, H., et al., The chemistry and applications of metal-organic frameworks. Science, 2013. 341(6149): p. 1230444.

19. Tranchemontagne, D.J., J.R. Hunt, and O.M. Yaghi, Room temperature synthesis of metal-organic frameworks: MOF-5, MOF-74, MOF-177, MOF-199, and IRMOF-0. Tetrahedron, 2008. 64(36): p. 8553-8557.

20. Long, J.R. and O.M. Yaghi, The pervasive chemistry of metal-organic frameworks. Chemical Society Reviews, 2009. 38(5): p. 1213-1214.

21. Davis, M.E., Ordered porous materials for emerging applications. Nature, 2002. 417(6891): p. 813-821.

22. Yim, C., et al., One-step immobilization of antibodies on ZIF-8/Fe 304 hybrid nanoparticles for the immunoassay of Staphylococcus aureus. RSC Advances, 2017. 7(3): p. 1418-1422.

23. Li, H., et al., Design and synthesis of an exceptionally stable and highly porous metal-organic framework. Nature, 1999. 402(6759): p. 276-279.

24. Eddaoudi, M., H. Li, and O. Yaghi, Highly porous and stable metal- organic frameworks: structure design and sorption properties. Journal of the American Chemical Society, 2000. 122(7): p. 1391-1397.

25. Yoo, Y. and H.-K. Jeong, Rapid fabrication of metal organic framework thin films using microwave-induced thermal deposition. Chemical communications, 2008(21): p. 2441-2443.

26. Choi, J.-S., et al., Metal-organic framework MOF-5 prepared by microwave heating: factors to be considered. Microporous and Mesoporous Materials, 2008. 116(1): p. 727-731.

27. Martinez Joaristi, A., et al., Electrochemical synthesis of some archetypical Zn2+, Cu2+, and Al3+ metal organic frameworks. Crystal Growth \& Design, 2012. 12(7): p. 3489-3498.

28. Stassen, I., et al., Solvent-free synthesis of supported ZIF-8 films and patterns through transformation of deposited zinc oxide precursors. CrystEngComm, 2013. 15(45): p. 9308-9311.

29. Cliffe, M.J., et al., Accelerated aging: a low energy, solvent-free alternative to solvothermal and mechanochemical synthesis of metal-organic materials. Chemical Science, 2012. 3(8): p. 2495-2500.

30. Farrusseng, D., S. Aguado, and C. Pinel, Metal-organic frameworks: opportunities for catalysis. Angewandte Chemie International Edition, 2009. 48(41): p. 7502-7513.

31. Yoo, Y., Z. Lai, and H.-K. Jeong, Fabrication of MOF-5 membranes using microwave-induced rapid seeding and solvothermal secondary growth. Microporous and Mesoporous Materials, 2009. 123(1): p. 100-106.

32. Bétard, A. and R.A. Fischer, Metal-Organic Framework Thin Films: From Fundamentals to Applications. Chemical reviews, 2011. 112(2): p. 1055-1083.

33. Ahrenholtz, S.R., C.C. Epley, and A.J. Morris, Solvothermal preparation of an electrocatalytic metalloporphyrin MOF thin film and its redox hopping charge-transfer mechanism. Journal of the American Chemical Society, 2014. 136(6): p. 2464-2472. 
34. Zhang, X., et al., New membrane architecture with high performance: ZIF-8 membrane supported on vertically aligned $\mathrm{ZnO}$ nanorods for gas permeation and separation. Chemistry of materials, 2014. 26(5): $p$. 1975-1981.

35. Yim, C., et al., Adsorption and desorption characteristics of alcohol vapors on a nanoporous ZIF-8 film investigated using silicon microcantilevers. Chemical Communications, 2015. 51(28): p. 6168-6171.

36. So, M.C., et al., Layer-by-layer fabrication of oriented porous thin films based on porphyrin-containing metal-organic frameworks. Journal of the American Chemical Society, 2013. 135(42): p. 15698-15701.

37. Shekhah, O., Layer-by-layer method for the synthesis and growth of surface mounted metal-organic frameworks (SURMOFs). Materials, 2010. 3(2): p. 1302-1315.

38. Hermes, S., et al., Selective nucleation and growth of metal- organic open framework thin films on patterned $\mathrm{COOH}$ /CF3-terminated self-assembled monolayers on Au (111). Journal of the American Chemical Society, 2005. 127(40): p. 13744-13745.

39. Makiura, R., et al., Surface nano-architecture of a metal-organic framework. Nature materials, 2010. 9(7): p. 565.

40. Ameloot, R., et al., Patterned growth of metal-organic framework coatings by electrochemical synthesis. Chemistry of Materials, 2009. 21(13): p. 2580-2582.

41. Zacher, D., et al., Deposition of microcrystalline [Cu 3 (btc) 2] and [Zn 2 (bdc) 2 (dabco)] at alumina and silica surfaces modified with patterned self assembled organic monolayers: evidence of surface selective and oriented growth. Journal of Materials Chemistry, 2007. 17(27): p. 2785-2792.

42. Hermes, S., et al., Selective growth and MOCVD loading of small single crystals of MOF-5 at alumina and silica surfaces modified with organic self-assembled monolayers. Chemistry of materials, 2007. 19(9): $p$. 2168-2173.

43. Goldberg, D.J., Current trends in intense pulsed light. J Clin Aesthet Dermatol, 2012. 5(6): p. 45-53.

44. Draper, G.L., et al., Fabrication of elemental copper by intense pulsed light processing of a copper nitrate hydroxide ink. ACS applied materials \& interfaces, 2015. 7(30): p. 16478-16485.

45. Hwang, Y.-T., et al., Intensive Plasmonic Flash Light Sintering of Copper Nanoinks Using a Band-Pass Light Filter for Highly Electrically Conductive Electrodes in Printed Electronics. ACS applied materials \& interfaces, 2016. 8(13): p. 8591-8599.

46. Kang, H., E. Sowade, and R.R. Baumann, Direct intense pulsed light sintering of inkjet-printed copper oxide layers within six milliseconds. ACS applied materials \& interfaces, 2014. 6(3): p. 1682-1687.

47. Singh, D.P., A.K. Ojha, and O.N. Srivastava, Synthesis of different $\mathrm{CU}(\mathrm{OH}) 2$ and $\mathrm{CuO}$ (nanowires, rectangles, seed-, belt-, and sheetlike) nanostructures by simple wet chemical route. The Journal of Physical Chemistry C, 2009. 113(9): p. 3409-3418.

48. Zhang, W., et al., Single-Crystalline Scroll-Type Nanotube Arrays of Copper Hydroxide Synthesized at Room Temperature. Advanced materials, 2003. 15(10): p. 822-825.

49. Li, J., et al., Zinc hydroxide nanostrands: unique precursors for synthesis of ZIF-8 thin membranes exhibiting high size-sieving ability for gas separation. CrystEngComm, 2014. 16(42): p. 9788-9791.

50. Chernikova, V., O. Shekhah, and M. Eddaoudi, Advanced Fabrication Method for the Preparation of MOF Thin Films: Liquid-Phase Epitaxy Approach Meets Spin Coating Method. ACS Applied Materials \& Interfaces, 2016. 8(31): p. 20459-20464.

51. Okada, K., et al., Copper Conversion into $\mathrm{Cu}(\mathrm{OH}) 2$ Nanotubes for Positioning Cu3 (BTC) 2 MOF Crystals: Controlling the Growth on Flat Plates, 3D Architectures, and as Patterns. Advanced Functional Materials, 2014. 24(14): p. 1969-1977.

52. Biesinger, M.C., et al., Resolving surface chemical states in XPS analysis of first row transition metals, oxides and hydroxides: Sc, Ti, V, Cu and Zn. Applied Surface Science, 2010. 257(3): p. 887-898.

53. Bradshaw, D., A. Garai, and J. Huo, Metal-organic framework growth at functional interfaces: thin films and composites for diverse applications. Chemical Society Reviews, 2012. 41(6): p. 2344-2381. 
54. Wang, M., et al., Rapid room-temperature synthesis of nanosheet-assembled ZnO mesocrystals with excellent photocatalytic activity. CrystEngComm, 2013. 15(4): p. 754-763.

55. Guo, H., et al., "Twin Copper Source" Growth of Metal- Organic Framework Membrane: Cu3 (BTC) 2 with High Permeability and Selectivity for Recycling H2. Journal of the American Chemical Society, 2009. 131(5): p. 1646-1647.

56. Bunzen, H., et al., From Micro to Nano: a toolbox for tuning crystal size and morphology of benzotriazolatebased metal-organic frameworks. Crystal Growth \& Design, 2016. 16(6): p. 3190-3197.

57. Ji, H., et al., Direct in Situ Conversion of Metals into Metal-Organic Frameworks: A Strategy for the Rapid Growth of MOF Films on Metal Substrates. ACS Applied Materials \& Interfaces, 2016. 8(47): p. 3241432420.

58. Tian, H., et al., Zeolitic imidazolate framework coated ZnO Nanorods as molecular sieving to improve selectivity of formaldehyde gas sensor. ACS Sensors, 2015. 1(3): p. 243-250.

59. Bux, H., et al., Zeolitic imidazolate framework membrane with molecular sieving properties by microwaveassisted solvothermal synthesis. Journal of the American Chemical Society, 2009. 131(44): p. 16000-16001.

60. Schejn, A., et al., Controlling ZIF-8 nano-and microcrystal formation and reactivity through zinc salt variations. CrystEngComm, 2014. 16(21): p. 4493-4500. 